\title{
Digital Image Processing of Urban Complex Thermal Environment Cao BaoShan ${ }^{1, a}$ \\ ${ }^{1}$ Chongqing College of Electronic Engineering, Chongqing, 401331, China \\ acbssx@163.com
}

Keywords: Urban complex; Thermal environment;Digitization;Mathematical model

\begin{abstract}
. the acceleration of global warming and urbanization progress has greatly contributed to the city building thermal environment problems, which causes the attention of people. However, because it involves many aspects, such as, it covers the architectural layout, building materials,trees greening,etc. In order to integrate the mutual influence of these factors into the study of whole complex outdoor thermal environment, this paper introduces the digital image processing technology, with the aid of its basic principle and certain image processing step, the influence factors of complex outdoor thermal environment are stored in digital form, so as to facilitate the analysis of complex outdoor thermal environment. Meanwhile, according to the different research content, this paper establishes various kinds of mathematical model, in order to get corresponding parameter values. Finally, it selects some examples to verify the mathematical model, and analyses the feasibility of urban complex thermal environment, then proves the rationality of mathematical model for complex outdoor thermal environment and correctness of the integration with image technology, which lay the foundation for the application of the model to complex outdoor thermal environment planning.
\end{abstract}

\section{Introduction}

Because there is the nature of complexity, integrity and dynamism in urban building outdoor environment, this paper takes building, ground, outdoor air and vegetation as the research object, including the parameters like air temperature, air humidity, underlying surface temperature,solar radiation and air velocity, and so on. In addition, influenced by the acceleration of global warming and urbanization process, the urban building outdoor thermal environment has a great change, there is certain difficulty in specific actual practice. In order to facilitate the study, according to the difference of pixel values, this paper uses digital image processing method to identify the information, such as architectural layout, building materials, greening distribution, water distribution, etc.,so that in the process of calculating modeling, the original information of physical model is retained in greatest extent, and the thermal physical parameters information of these factors is integrated into outdoor thermal environment model of whole residential area, facilitating the further analysis and study.

\section{Overview of digital image processing method}

Digital image processing is the process of using computer to process image, from the angle of content, it can be divided into image digitization, image transformation, image enhancement, image restoration, image segmentation, image analysis and image compression, etc., it is widely used in various fields. In this article, the digital image processing used is the fourth generation computer language MATLAB. The digital image in MATLAB is represented by one or more matrix. In MaTLAB, the image is stored as pixel, namely each element of matrix represents a pixel, for example,a pair of image with 200 lines and 300 columns, which is stored as matrix of $200 \times 300$ size. The concrete implementation of digital image processing needs certain steps, in order to get digital information of urban complex underlying surface image distribution. The specific steps are shown in figure 1: 


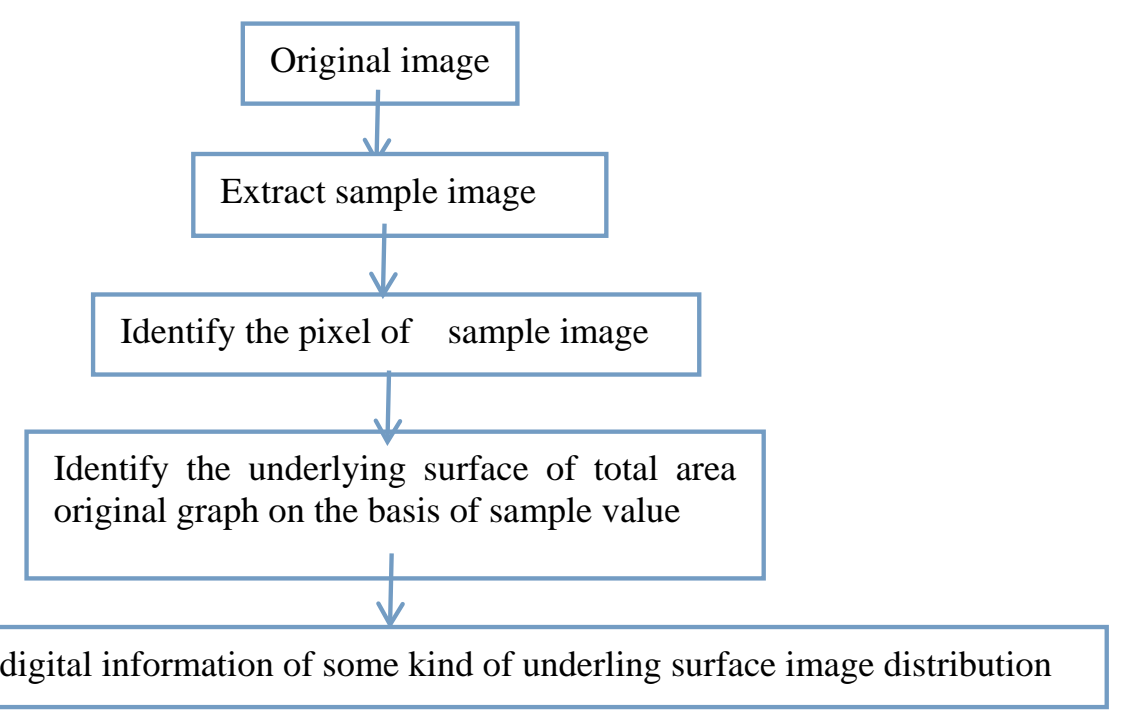

Obtain the digital information of some kind of underling surface image distribution

Figure 1 Digital image processing flow diagram

At the same time, as for the storing of data file, most of the image type belong to the true color image (RGB)and gray-scale image. The so-called RGB image mainly use red, green and blue to represent one kind of pixel color respectively, according to the image recognition of actual environment, it can be composed any color. The use of three-dimensional matrix shows that each dimension represents a kind of color, so this RGB image with 200 lines and 300 columns will use the matrix of $200 \times 300 \times 3$ size. Therefore, for a RGB image with $m \times n$ size, it should be stored as multidimensional matrix of $m \times n \times 3$ size in MATLAB. It is shown in figure 2 :

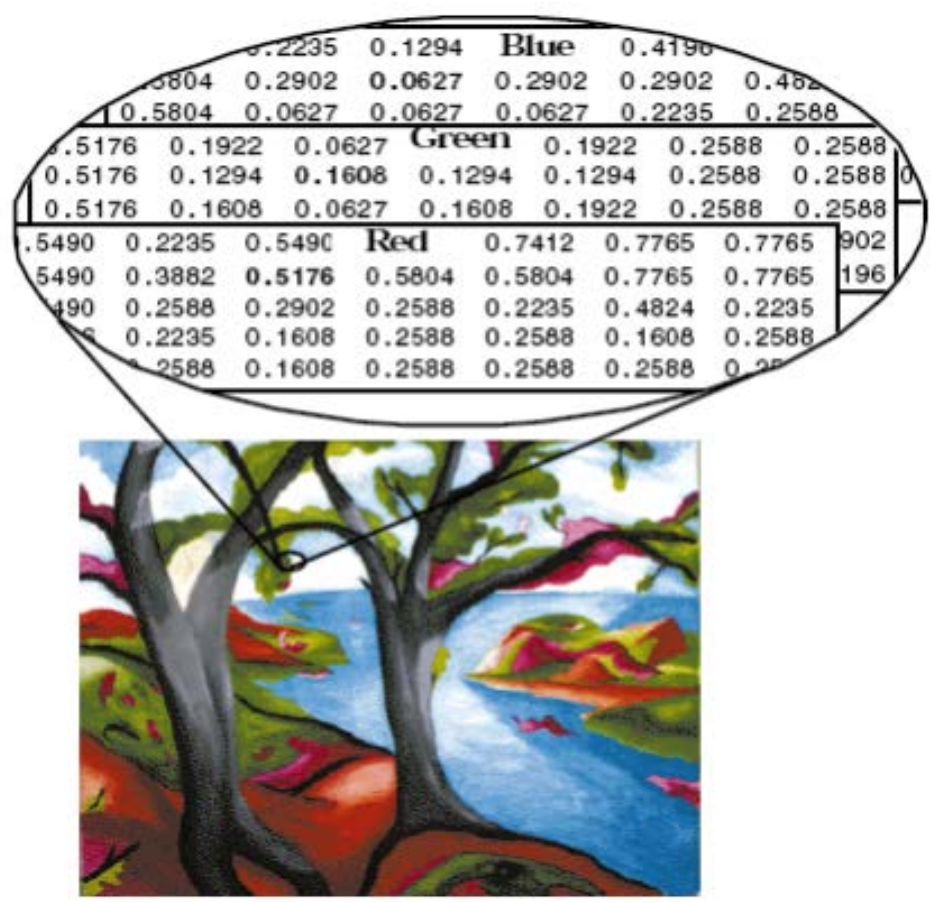

Figure 2 Digital storage format of RGB

However, the gray-scale image shows only intensity information, it only needs a digital matrix, each element of matrix represents gray value of pixel in corresponding location, in actual operation, based on the data type function transfer, the basic image information is reflected in simple black and white color, which is a simple way of data file storage. 


\section{Mathematical modeling of urban complex thermal environment}

After obtaining the material distribution of urban building underlying surface by image recognition, only the thermal physical property parameters of different material are further got, we can accurately understand the parameter distribution of material density, specific heat and coefficient of heat conductivity in the whole image area, which is then stored in different pixel mathematical matrix of $200 \times 200$ size, at last this paper uses outdoor thermal environment mathematical model to make a detailed analysis.

\section{Air flow mathematical model in complex}

Using an actual urban complex as the research object, its layout and the regional distribution of flow is shown as figure3, figure 4, then marking number on different buildings, so as to distinguish them.

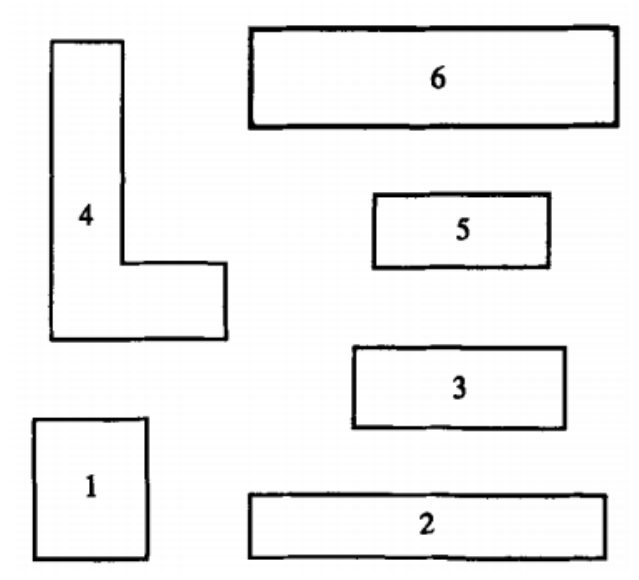

Figure 3 Layout of urban complex

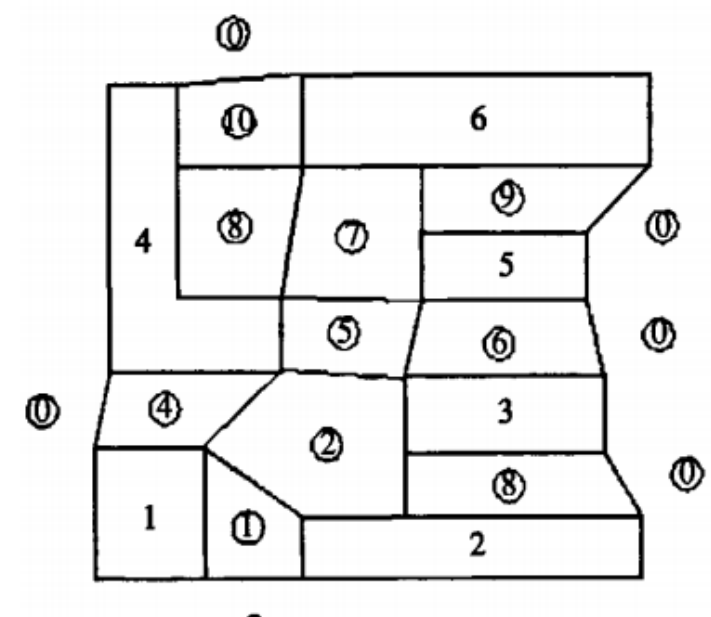

(1)

Figure 4 Diagram on the flow region division of urban complex

After knowing the city complex distribution and flow regional division, we can constitute rules according to the graph theory, and abstract flow area, obtain the network diagram of flow area. Ventilation circuit is used to connect matrix and express the connection relationship between each node and branch. The line of matrix is sorted as the node number, the column is sorted as the branch number. Each element value of the matrix is +1 , l, or 0 . For some node $i$, if the branch $j$ flows into the node, its corresponding element(i,j) value is -1 ; whereas, if the node outflow, the element(i,j) value is +1 ; If node has nothing to do with branch, the element(i,j) value is 0 . In this way, the complex ventilation circuit is constituted to connect matrix formally, facilitating the establishment 
of flow area mass conservation equations set.

When calculating the mass balance of some flow area i, it can be performed by equation (1).

$$
M_{\mathrm{i}}=V_{\mathrm{i}} \frac{\partial \rho_{\mathrm{i}}}{\partial \tau}+\sum_{\mathrm{j}}\left(\mathrm{m}_{\mathrm{ij}}-\mathrm{m}_{\mathrm{ji}}\right)
$$

$\mathrm{V}_{\mathrm{i}}$ in above equation refers to the volume of region $\mathrm{i}, \rho_{\mathrm{i}}$ refers to air density, $\mathrm{m}_{\mathrm{ij}}$ represents air flow from region $i$ to $j, m_{i j}$ represents air flow from region $j$ to $i, M_{i}$ represents the air quality occurrence amount of region i.Combined with the city building flow regional distribution, the mass balance equation of all flow region is established, after ignoring

small flow rate of the first item in equation, the below equation is got:

$$
M_{\mathrm{i}}=\sum_{\mathrm{j}}\left(\mathrm{m}_{\mathrm{ij}}-\mathrm{m}_{\mathrm{ji}}\right)
$$

Combined with formal connection matrix of complex ventilation circuit and a variety of calculation correlation, the pressure balance equation of complex area flow network loop, flow opening pressure differential, flow relationship equation and the flow area air temperature heat balance equation are set up, the specific formula is shown as follows:

$$
\sum_{\mathrm{i}} P_{\mathrm{i}}=\sum_{\mathrm{i}}\left(P_{\mathrm{hi}}+P_{\mathrm{si}}\right)=0
$$

The above formula (3) is the pressure balance equation of complex area flow network loop, and $\mathrm{P}_{\mathrm{i}}$ refers to total pressure differential algebraic sum of flow openings $\mathrm{i}, \mathrm{P}_{\mathrm{hi}}$ refers to pressure differential of flow openings $i, P_{s i}$ is pressure source of opening $i$.

According to Bernoulli's equation, it can be derived that the air density of flow openings on both ends depends on the pressure differential on both sides, and the openings flow calculation formula is as follows:

$$
\mathrm{m}=\mu \mathrm{A} \sqrt{2 \rho P}
$$

After the further difference, it can be learned about the opening pressure differential of $\mathrm{P}$ in $\mathrm{n}+1$ time:

$$
P_{\mathrm{n}+1}=P_{\mathrm{n}}-\frac{\mathrm{m}_{\mathrm{n}}^{2}}{\mu^{2} A^{2} \rho}+\frac{\mathrm{m}_{\mathrm{n}}}{\mu^{2} A^{2} \rho} \mathrm{m}_{\mathrm{n}+1}
$$

And the air heat balance in urban complex area is shown as figure 5, the air heat balance equation set up in the area is as follows:

$$
Q_{\mathrm{i}}=Q_{F \cdot \mathrm{i}}+Q_{C \cdot \mathrm{i}}
$$

At the same time, $Q_{F \cdot \mathrm{i}}$ and $Q_{C \cdot \mathrm{i}}$ in the above formula refer to their algebraic sum in all path, that is:

$$
\begin{gathered}
Q_{F \cdot \mathrm{i}}=\sum_{\mathrm{j}} Q_{F \cdot \mathrm{ij}} \\
Q_{C \cdot \mathrm{i}}=\sum_{\mathrm{k}} Q_{C \cdot \mathrm{ik}}
\end{gathered}
$$



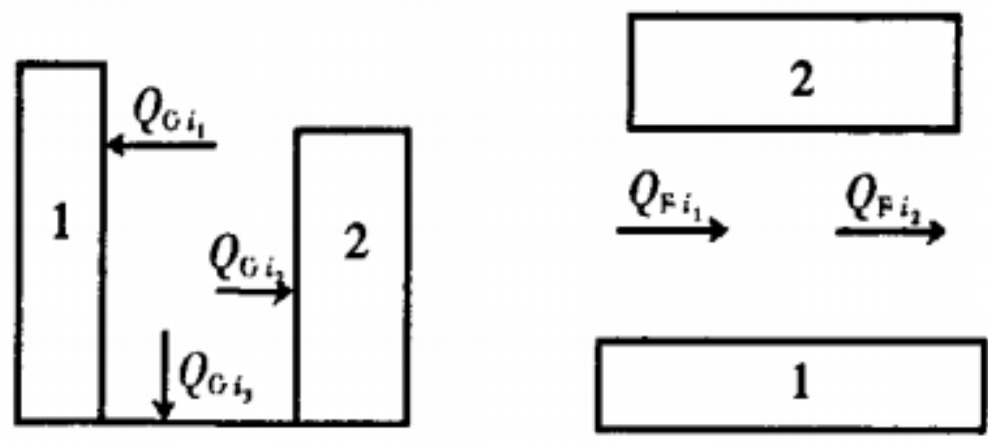

Figure 5 Air heat balance diagram in urban complex region

\section{Analysis on building facades structure and ground heat transfer}

Through the observation about building facades wall body structure and ground heat transfer model in figure 6 and figure 7, the corresponding calculation equations can be obtained.

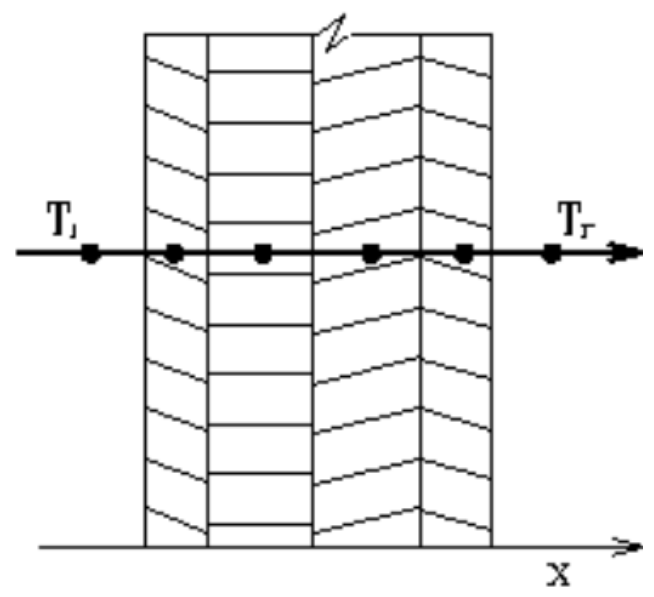

Figure 6 Diagram for building facades wall body structure

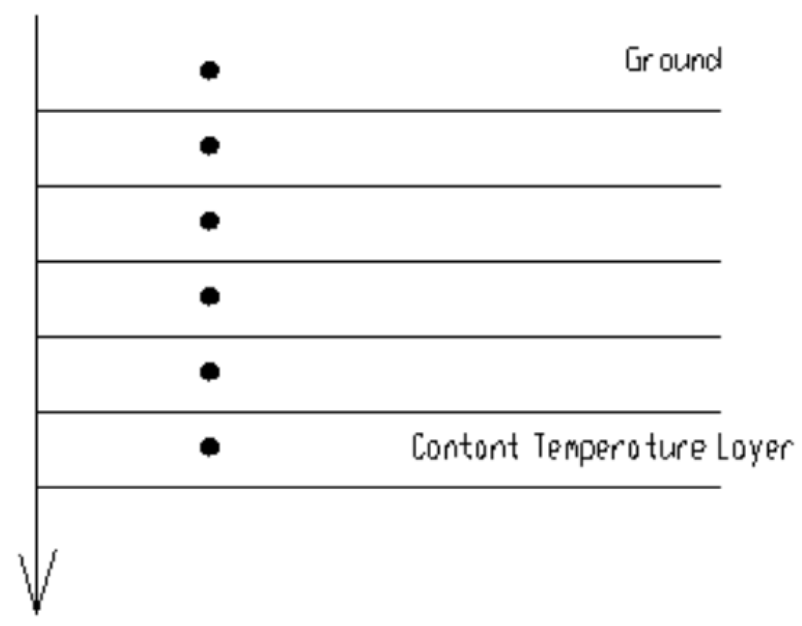

Figure 7 Ground-based heat transfer model

Combined with the wall structure in figure 6, the one-dimensional heat conduction equation used in its heat transfer is as follows:

$$
\rho C \frac{\partial T}{\partial \tau}=\frac{\partial}{\partial \chi}\left(\lambda \frac{\partial T}{\partial \chi}\right)
$$


According to the calculation method of boundary condition, under the guidance of ground heat-transfer model, it is calculated that if $x=0$, the boundary condition of complex ground surface heat conduction is as follows:

$$
-\lambda \frac{\partial T}{\partial \chi}=\alpha_{\mathrm{ik}}\left(T_{\mathrm{i}}-T\right)+(1-\rho) \cdot\left(Q_{D}+Q_{B}+Q_{L}+Q_{A}\right)-Q_{S}
$$

Certainly, in combination with other specific research objects of urban complex thermal environment, the parameters like air humidity, solar radiation and air velocity can be obtained through the effective combination of digital image processing and mathematical model, which further ensuring the comprehensive scientific rationality of urban complex thermal environment research. Considering that the problem of space limitation, this article will not discuss one by one.

Through the above established air flow mathematical model inside complex, building facades structure and ground heat-transfer calculation equation, the specific data information of certain urban complex thermal environment is obtained, which must be validated by examples, and then making a comparison with the measured results, ensuring the rationality of the mathematical model. By the comparison between air temperature of the simulated flow area 3 in a certain city complex and measured results, it is got that the results are approximate, proving the rationality of mathematical model construction for complex thermal environment.

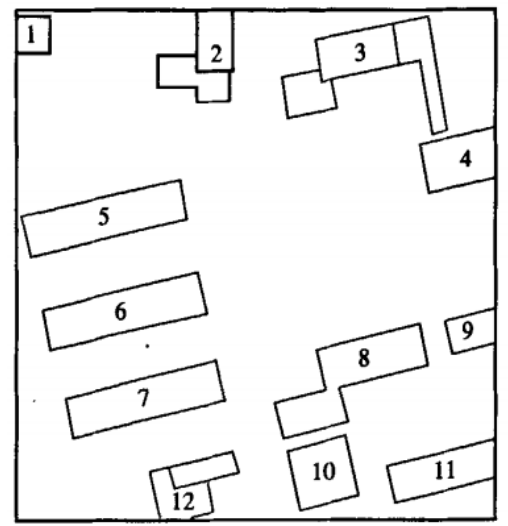

Figure 8 Diagram for specific distribution of urban complex and building number

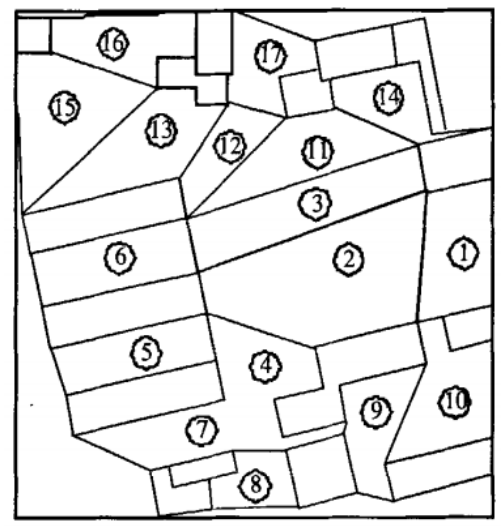

Figure 9 Diagram for flow region division in measured model 


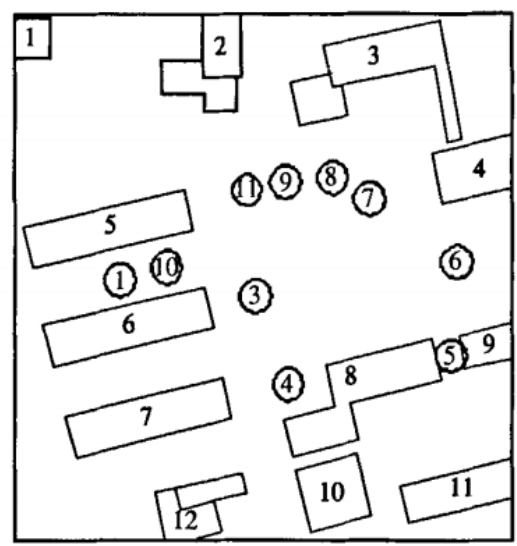

Figure 10 Diagram for point location in measured model

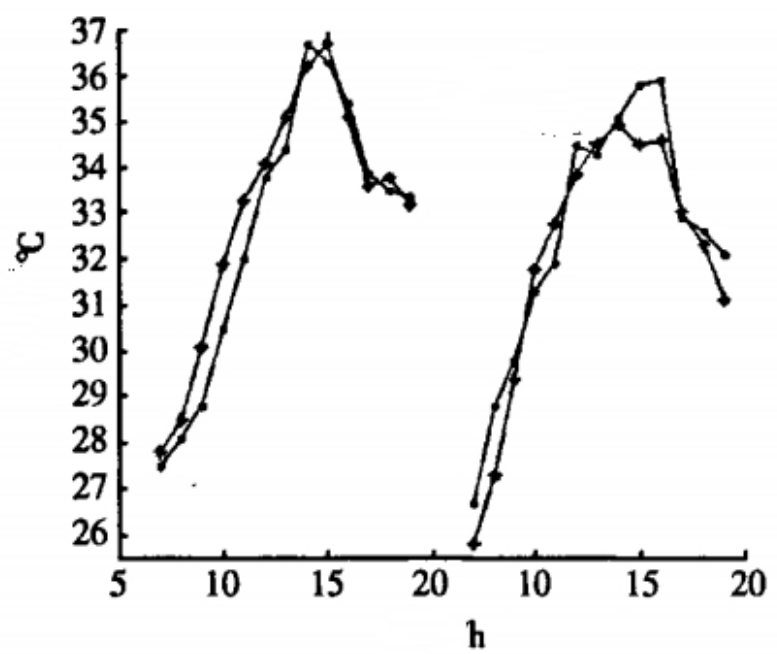

Figure 11 Diagram for air temperature comparison in region 3

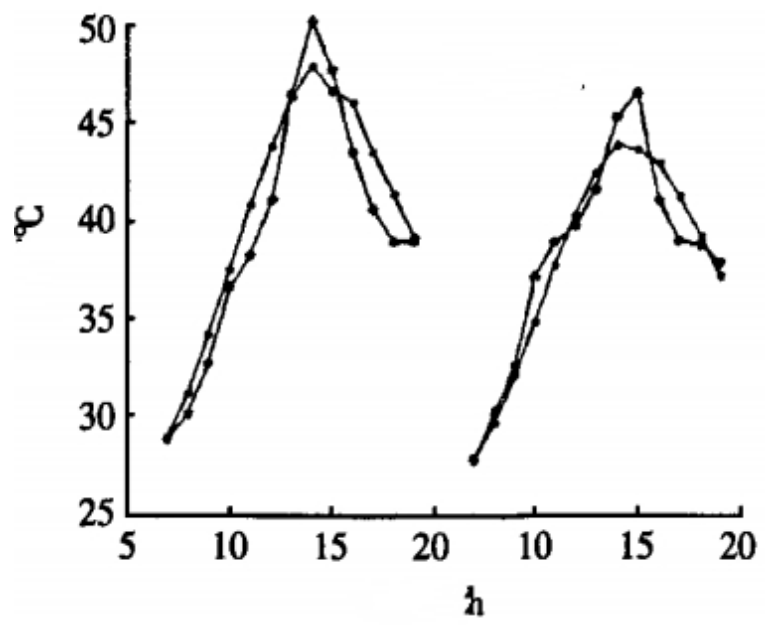

Figure 12 Diagram for ground temperature comparison in point 3

( note: -1 represents test values,

represents analog value )

\section{Conclusion}

To sum up, in the process of studying urban building thermal environment, this paper introduces digital image processing technology, builds the air flow area network model, heat conduction image analysis model, then on this basis, this article takes building, ground, air as object and builds energy balance mathematical model for air heat convection, heat conduction, radiation, 
so as to get the related parameters data effectively, which is similar with the experimental result, therefore it has higher practical application value.

\section{Knowledgements}

Project Name1: applicability and experimental study of cooling and evaporative cooling technology in rural areas of Chongqing

Project unit: Science and technology research project of Chongqing Municipal Education Committee

Item number: KJ1602911

Project Name2: data driven predictive control based on subspace identification

Project unit: Science and technology research project of Chongqing Municipal Education Committee

Item number: KJ1503008

\section{Reference}

[1] Luoqing, Yu Jiuyi. Analysis Method on Urban Residential Area Outdoor Thermal Environment[J]. Civil and environmental engineering, 2013,35(1): P147-152

[2] Luoqing. Study on Urban Complex Outdoor Thermal Environment Based on Image Analysis[D]. $\mathrm{PhD}$ dissertation, Chongqing University,2006

[3] Luoqing, Linan. Digital Graphic Analysis on Urban Complex Outdoor Thermal Environment[Z]. Academic Seminar on Air Conditioning Simulation Analysis of National Hvac Professional Committee, 2005: P 349-356

[4] Yuan Yongdong. Analysis on the Influence of Different Construction Layout on Outdoor Thermal Environment and Energy-Saving Effect[D]. Master's thesis, Donghua University, 2011

[5] Luoqing, Li Baizhan. Mathematical Model of Complex Outdoor Thermal Environment Based on Image Processing and Example Analysis [J]. Journal of Solar Energy, 2008,29（5） : P 630-636

[6] Hu Xiaojun, Tang Xiaomin. Simulation Study on Urban Residential Area Outdoor Thermal Environment[J]. Building Science, 2012 (s2): P 260-265

[7] Gao Yafeng. Measured and Simulation Study on Outdoor Thermal Environment Suitable for Urban Residential Area Planning[D]. PhD dissertation, Chongqing University,2011

[8] Shao Yuling. Simulation Study on Outdoor Thermal Environment Values in Urban Residential Area[D]. Master's thesis, Zhejiang University, 2012 